\title{
News from the isolated ellipticals NGC 5812, NGC 7507, and NGC 7796
}

\author{
Tom Richtler ${ }^{1}$, Ricardo Salinas ${ }^{2}$, Richard Lane ${ }^{1}$, Michael Hilker ${ }^{3}$, \\ Juan Pablo Caso ${ }^{4}$ and Lilia P. Bassino ${ }^{4}$ \\ ${ }^{1}$ Departamento de Astronomía, Universidad de Concepción, Chile \\ ${ }^{2}$ Department of Physics and Astronomy, Michigan State University, East Lansing, Michigan, \\ USA \\ ${ }^{3}$ European Southern Observatory, Garching, Germany \\ ${ }^{4}$ Universidad Nacional de La Plata and IALP (CONICET-UNLP), La Plata, Argentina
}

\begin{abstract}
We report on ongoing photometric and spectroscopic work on a sample of isolated elliptical galaxies. We investigate their globular cluster systems, and use the kinematics of globular clusters and the integrated galaxy light to constrain their dark halos, which are not found in the cases of NGC 5812 and NGC 7507.
\end{abstract}

Keywords. galaxies: elliptical and lenticular, cD - galaxies: evolution - galaxies: formation galaxies: halo

\section{Introduction}

Isolated galaxies are expected to evolve differently from galaxies in groups or clusters. This should be manifest particularly in the structures of their visible and dark halos, where globular clusters (GCs) can be tracers for both components. We collected photometric data, using VLT, Gemini South, and $4 \mathrm{~m}$ Blanco telescope, of a dozen isolated ellipticals. Besides characterising their GC systems, we want to identify clusters suitable for the use as dynamical tracers for testing cosmological simulations (Niemi et al. 2010).

\section{NGC 5812 - alone in the dark?}

NGC 5812 (distance $28 \mathrm{Mpc}$ ) is a galaxy with a strong intermediate-age stellar component. The accompanying dwarf galaxy at a projected distance of about $20 \mathrm{kpc}$ shows an extended tidal tail. Photometric data come from the 4m-Blanco telescope. Lane et al. (2013) describes its morphology, photometric properties and cluster system, which is rather poor. We obtained mask spectroscopy with GMOS/Gemini-South (GS-2013BQ51; PI: Richtler) and got radial velocities for $25 \mathrm{GCs}$ out to a projected radius of 20 kpc. The brightest object has about $M_{R} \approx-12$, confirming the existence of clusters of this brightness as statistically predicted in the CMD shown by Lane et al. One cluster has a strongly deviating velocity of $1440 \mathrm{~km} / \mathrm{s}$ (systemic velocity $1970 \mathrm{~km} / \mathrm{s}$ ), while 24 objects show a tight distribution around the systemic velocity of NGC 5812 with a dispersion of only $95 \mathrm{~km} / \mathrm{s}$. This low value is puzzling. A sample of 24 GCs should already permit a reliable estimation of the total velocity dispersion of the cluster system. The central velocity dispersion of $200 \mathrm{~km} / \mathrm{s}$ (HyperLeda) requires with our photometric model a stellar $M / L_{R}$-value of about 3.5 under isotropy. While we await a more complete dynamical analysis, we anticipate that it will be difficult to reconcile the low velocity dispersion with the existence of a massive dark halo. NGC 5812 thus seems to be another candidate among isolated ellipticals for having less dark matter than expected. 


\section{NGC 7507 - little dark matter}

NGC 7507 (distance $27 \mathrm{Mpc}$ ) belongs to the galaxies with a Keplerian decline of the projected velocity dispersion (Salinas et al. 2012). It also has a quite poor GC system (Caso et al. 2013) and we could not use GCs as dynamical tracers. The kinematic data within 1 arcmin stem from long-slit observations. By deep mask spectroscopy, obtained with Gemini/GMOS (GS-2009B-Q84; PI: Salinas) we extended the radius with measured velocity dispersions out to 130 arcsec (Lane et al. 2014). The adjacent regions agree excellently, but at 70 arcsec, we observe a bump in the velocity dispersion profile, which according to Schauer et al. (2014) might be the relic of a former merging event. At larger radii, the velocity dispersion profile is again well described by the stellar mass alone. Radial anisotropy and the inclusion of rotation may help to accommodate some dark matter.

\section{NGC 7796 - an isolated cluster elliptical}

Deep imaging with VLT/VIMOS in B and R (89.B-457; PI: Salinas) builds the database for our investigation of the GC system of NGC 7796 (distance $50 \mathrm{Mpc}$ ), which is a massive old galaxy without striking substructure. However, a companion dwarf galaxy shows tidal tails and multiple nuclei/star cluste bluer than their parent galaxy. In contrast to many other isolated ellipticals, the GC system of NGC 7796 with estimated 2000 members rivals some cluster ellipticals. We derive a specific frequency of $S_{N}=2.6 \pm 0.5$. We see the familiar bimodal distribution in B-R which characterises an old cluster system. The density profiles of blue and red GCs agree within the uncertainties. Therefore the growth of the halo probably did not happened through later accretion of dwarf galaxies, as expected for an isolated elliptical. More insight will come from comparing the kinematics of metal-poor and metal-rich GCs. The kinematical literature data for the galaxy centre result in $M / L_{R}=6.5$ for the stellar component under isotropy, consistent with an old metal-rich population, but do not permit solid statements regarding the dark matter content. Assuming MOND, $M / L_{R}$ becomes 6.2, and the X-ray data of O'Sullivan et al. (2007) are consistent with the MONDian prediction. In comparison with NGC 7507 and NGC5812, NGC7796 may indicate that besides accretion, the epoch of star formation determines the richness of a GC system.

\section{Acknowledgements}

TR acknowledges support from FONDECYT project Nr. 1100620, the BASAL Centro de Astrofísica y Tecnologías Afines (CATA) PFB-06/2007, and a visitorship at ESO/Garching.

\section{References}

Caso J. P., Richtler T., Bassino, L. P., et al. 2013, A\&A A, 555, 56

Lane R., Salinas R., \& Richtler, T. 2013, A\& A, 549, 148

Lane R., Salinas R., \& Richtler, T. 2014, $A \mathscr{E} A$, accepted

Niemi S., Nurmi P., Heinämäcki, P., \& Saar, E. 2010, MNRAS, 344, 1000

O'Sullivan E., Sanderson A. J. R., \& Ponman, T. 2007, MNRAS, 380, 1409

Richtler T., Salinas R., Lane R., Hilker M., \& Schirmer M. 2014, A\&AA, submitted

Salinas R., Richtler T., Bassino, L. P., et al. 2012, A\&4A, 538, A87

Schauer A. T. P.h., Remus R. S., Burkert, A., \& Johansson P. H. 2014, ApJ, 783, 32 